\title{
A New Model of Electromechanical Relays for Predicting the Motion and Electromagnetic Dynamics
}

\author{
Edgar Ramirez-Laboreo, Student Member, IEEE, Carlos Sagues, Senior Member, IEEE, and Sergio Llorente
}

\begin{abstract}
In this paper, a novel multiphysics and nonlinear model for electromechanical relays is presented. The electromagnetic dynamics is analyzed by calculating the total reluctance of the magnetic equivalent circuit, which is composed of a fixed length iron core and an angular air gap. Magnetic saturation and angular dependency of the reluctance are considered in the analysis. Then, an energy balance over the electromagnetic components of the system is used to obtain the torque which drives the movable armature. A planar mechanism of four rigid bodies, including spring-damping torques that restrict the motion and model the contact bounces that occur in the switchings, is proposed to explain the dynamics of the movable components. Experimental tests show the accuracy of the model both in the electromagnetic and the mechanical parts.
\end{abstract}

Index Terms-Electromechanical devices, switches, relays, contactors, modeling, magnetic equivalent circuit, planar mechanism, contact bounce.

\section{INTRODUCTION}

$\mathbf{E}$ LECTROMECHANICAL devices such as relays or contactors are widely used in domestic and industrial applications. Although their operating principle comes from the nineteenth century, these devices still offer many advantages over their semiconductor counterparts: they have lower energy losses, are able to conduct and block current in both directions, do not require drivers to be activated and are slightly cheaper. For these reasons, they are extensively used in products needing some type of electric or electronic regulation. Relays and contactors can be found, e.g., in cars, home appliances, communications systems or industrial machines. Despite these advantages, electromechanical devices also present some drawbacks related to their operation mode. They are considerably slower than solid-state switches and, since there are moving components, they are continuously subject to wear and their service life is shorter. Additionally, strong bounces are generated in each switching when the movable parts hit the fixed ones, originating additional wearing and a noise which is a problem in many applications. As a result, electromechanical devices are often discarded in favor of solid-state switches.

In order to reduce the problems and increase the benefits, several research groups have been working on models and control strategies for these electromechanical devices. One of

E. Ramirez-Laboreo and C. Sagues are with the Departamento de Informática e Ingeniería de Sistemas (DIIS) and the Instituto de Investigación en Ingeniería de Aragón (I3A), Universidad de Zaragoza, Zaragoza 50018, Spain.e-mail: ramirlab@unizar.es (E. Ramirez-Laboreo), csagues@unizar.es (C. Sagues).

S. Llorente is with the Research and Development Department, Induction Technology, Product Division Cookers, BSH Home Appliances Group, Zaragoza 50016, Spain. e-mail: sergio.llorente@bshg.com the very first attempts to study the contact bounce phenomenon was [1], where an energy-based analysis and some simple spring-mass systems were proposed to explain the process dynamics. A good review of electrical contacts including the influence of the arc current was made years later [2], and one of the first works regarding the control of contact bounce was presented in [3]. With respect to modeling, an early mechanical and electromagnetic coupled model including contact bounce was already presented in [4], but the authors themselves suggested that some aspects should be studied deeply.

Many researches have been presented in recent years for predicting the electromagnetic dynamics and the motion of relays and contactors. Different approaches have been considered for the electromagnetism, ranging from models with low computing requirements based on Magnetic Equivalent Circuits (MEC) to comprehensive and time consuming Finite Element Method (FEM) analyses. Concerning the first approach, a detailed framework for building three-dimensional electromagnetic MEC-based models can be found in [5]. An electromagnetic MEC model for AC contactors including magnetic saturation is proposed in [6], while the motion dynamics and a position estimator are presented by the same authors in a previous paper [7]. A MEC model is also used in [8] to optimize the geometry of a permanent-magnet contactor. Some works combining the two methods can also be found. See, e.g., the research in [9], where a MEC-based model is improved by using a correction factor calculated from FEM simulations, or [10], where an one-dimensional MEC model and a two-dimensional FEM analysis are compared. On the other hand, pure FEM-based models have been proposed, e.g., for calculating the attractive torque of a permanent-magnet relay [11]. In spite of being very common in the market, only a few works [9], [11] present models for electromechanical switches with angular air gaps. To the best knowledge of the authors, there is no work that fully analyzes this type of devices from the MEC approach. Different options have been considered also for the motion. A mechanical model based on the Euler-Bernoulli theory for beams is proposed to predict the motion of a relay in [12], where a viscoelastic spring-damper model is also presented for explaining the contact bounces. The beam theory is used in other researches [10], but the most widespread approach is the use of rigid-body models with rectilinear motions and a single degree of freedom [4], [7], [8].

Some research has also been published regarding the control of electromechanical devices. Nevertheless, the problem is still not satisfactory solved because of the highly nonlinear dynamics and the partially stochastic behavior of these devices. Certain works propose, e.g., position controllers based on non- 
linear estimators [7], [13] or intelligent methods [8]. In [14], a control algorithm that limits the current through the coil of a contactor is described for reducing contact bounce. Control strategies can be found also for other electromechanical devices, e.g., for an electromagnet excited pendulum [15] or for a solenoid valve [16], but they are always dependent on a dynamic model of the system. Considering the large amount of electromagnetic switches in the market [17], the need for new and better dynamic models becomes evident.

The research on electromechanical relays and contactors has not been limited to predicting their motion or electromagnetic dynamics. See, e.g., the method proposed in [18] to evaluate the erosion of the electrical contacts of a contactor, the FEM-based thermal model of sealed electromagnetic relays developed in [19], the lifetime statistical analysis under various temperature conditions in [20], the endurance prediction model for ac relays in [21] or the quality analysis presented in [22]. Besides, novel dynamic models are also being developed for other types of electromechanical switches [23].

In this paper, we present a new MEC-based model for electromechanical relays with the following contributions: (1) it describes the electromagnetic dynamics of a device with an angular air gap taking into account the magnetic saturation of the iron core, (2) it studies the motion of a complex mechanism by means of a rigid-body model with two degrees of freedom, (3) it allows one to analyze the contact bounces generated in the closure and the opening by means of spring-damping torques and (4) it requires a much lower computational cost than FEM-based models. An earlier version of this paper was already presented at the 2015 IAS Annual Meeting [24].

The paper is structured as follows. First, in section II, the electromechanical relay investigated in this work is presented and its operating mode explained. The electromagnetic dynamics of the system is described in section III by calculating the reluctance of the magnetic equivalent circuit. Besides, an analysis based on the electromagnetic energy is made to obtain an expression for the torque that drives the motion of the relay. Then, in section IV, a planar mechanism composed of four rigid bodies is proposed to describe the motion of the movable components. This mechanical model includes articular limits so that the motion of the mechanism is restricted to specific positions. These bounds are achieved by means of springdamping torques that are also able to model the contact bounces that usually exist in the operation of electromechanical devices. Experimental tests have been carried out to validate the model and the results are presented and discussed in section V. Finally, the conclusions of the research are presented in section VI.

\section{SYSTEM DESCRIPTION}

The electromechanical switch used in this research is a general purpose PCB power relay which features a high electrical insulation between the coil and the contacts (Fig. 1). This is achieved by means of a mechanism with an intermediate plastic part that completely separates the electromagnet and the power terminals. It is single pole and double throw, and similar models are sold by the main manufacturers in the market. Its operating mode is as follows. When the coil is not energized, the movable contact stands still, touching the normally closed contact. If the coil is energized, the movable armature closes the magnetic circuit and pushes the plastic part. At the same time, the opposite end of the plastic component causes the movable contact to touch the normally open contact. This process is called making or closing. When the coil is deenergized, elastic forces make the relay return to its original position, opening the magnetic circuit and closing the normally closed connection. This second process is called breaking or opening.

\section{ELECTROMAGNETIC MODEL}

\section{A. Current and magnetic flux dynamics}

The electromagnetic components of the relay are schematically represented in Fig. 2(a). The coil has $N$ turns and electrical resistance $R$. The magnetic circuit is composed of a soft iron core, with a fixed and a movable part, and an air gap. The geometry of the core is known and the size of the air gap is determined by the angular position of the movable armature $\theta_{1}$. When a voltage difference $v$ is applied between the coil terminals, the electrical behavior of the system is given by

$$
v=R i+N \frac{\mathrm{d} \phi}{\mathrm{d} t},
$$

where $i$ is the current flowing through the coil and $\phi$ is the magnetic flux through the core and the air gap. Current $i$ and magnetic flux $\phi$ are also related by means of the equation of the magnetic equivalent circuit,

$$
N i=\phi \Re,
$$

where $\Re$ is the total reluctance of the circuit and can be divided into the air gap reluctance $\Re_{g}$ and the magnetic core reluctance $\Re_{c}$ (Fig. 2(b)).

$$
\Re=\Re_{g}+\Re_{c} .
$$

The first component of $\Re$ is calculated from the geometry of the air gap,

$$
\Re_{g}=\frac{l_{g}}{\mu_{0} A_{g}}=\frac{r_{g} \theta_{1}}{\mu_{0} A_{g}},
$$

where $l_{g}$ and $A_{g}$ are the length and the cross-sectional area of the air gap, $r_{g}$ the average turning radius of the crosssection, $\theta_{1}$ the rotation angle of the moving armature and $\mu_{0}$ the vacuum magnetic permeability.

On the other hand, the reluctance of the magnetic core is approximated by using an average path length $l_{c}$ and an average cross-sectional area $A_{c}$ of the magnetic core,

$$
\Re_{c}=\frac{l_{c}}{\mu_{c} A_{c}},
$$

where $\mu_{c}$ is the magnetic permeability of the core material. The relationship between magnetic flux density $B_{c}$ and magnetic field intensity $H_{c}$ within the magnetic core is considerably nonlinear due to magnetic saturation. Consequently, $\mu_{c}$ cannot be considered constant. To reflect this behavior, an alternative version of the Froelich equation has been used,

$$
B_{c}=\frac{c_{1} H_{c}}{1+c_{2}\left|H_{c}\right|},
$$




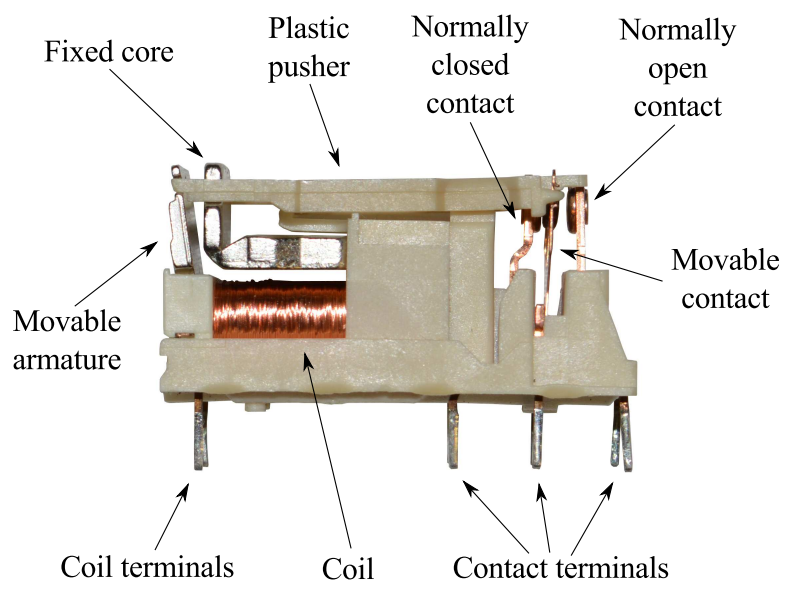

Fig. 1. Electromechanical relay.

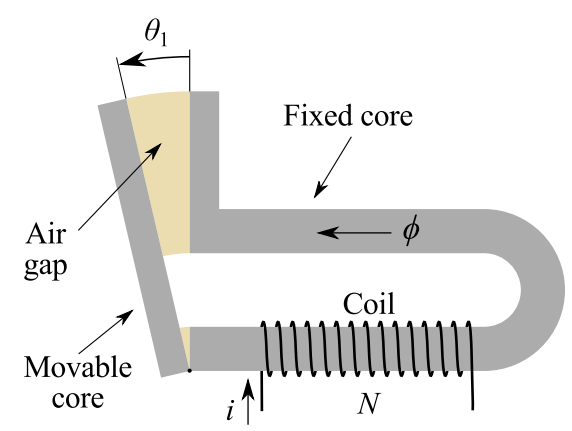

(a)

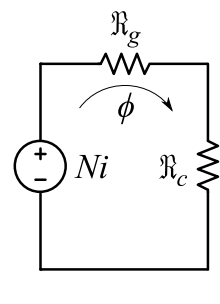

(b)
Fig. 2. (a) Scheme of the electromagnetic components and (b) Magnetic equivalent circuit.

where $c_{1}$ and $c_{2}$ are constants which have been experimentally fitted. Although similar expressions have been already used in previous works [6], [7] with good results, it must be noted that the absolute value included in the denominator allows for the calculation also with negative values of $H_{c}$. Otherwise, meaningless values of $\mu_{c}$ would be obtained and divisions by zero may appear in the equations.

According to (6), $\mu_{c}$ may be expressed either as a function of magnetic field $H_{c}$ or of magnetic flux density $B_{c}$.

$$
\mu_{c}=\frac{B_{c}}{H_{c}}=\frac{c_{1}}{1+c_{2}\left|H_{c}\right|}=c_{1}-c_{2}\left|B_{c}\right| .
$$

Considering that $\phi=B_{c} A_{c}$, the previous expression is transformed to be in terms of magnetic flux.

$$
\mu_{c}=c_{1}-\frac{c_{2}}{A_{c}}|\phi| \text {. }
$$

Now, by substituting (8) in (5) and then in (3), the expression for the total reluctance of the magnetic circuit is obtained.

$$
\Re=\frac{r_{g} \theta_{1}}{\mu_{0} A_{g}}+\frac{l_{c}}{c_{1} A_{c}-c_{2}|\phi|} .
$$

Note that reluctance $\Re$ depends on angular position $\theta_{1}$ and magnetic flux $\phi$, hence including the variations caused by the angular motion of the armature and by magnetic saturation. For simplicity in the notation, (9) will be expressed from now on as

$$
\Re=k_{1} \theta_{1}+\frac{k_{2}}{1-k_{3}|\phi|},
$$

where

$$
k_{1}=\frac{r_{g}}{\mu_{0} A_{g}}, \quad k_{2}=\frac{l_{c}}{c_{1} A_{c}}, \quad k_{3}=\frac{c_{2}}{c_{1} A_{c}} .
$$

Substituting (10) in (2), and solving the system of (1) and (2), the explicit equation for the dynamics of the magnetic flux is finally obtained.

$$
\frac{\mathrm{d} \phi}{\mathrm{d} t}=\frac{v}{N}-\frac{R \phi}{N^{2}}\left(k_{1} \theta_{1}+\frac{k_{2}}{1-k_{3}|\phi|}\right) .
$$

Now, if angular position $\theta_{1}$ is known, (11) can be solved by numerical integration and then the current through the coil is directly obtained by using (2) and (10). In addition, inductance $L$ of the coil can also be calculated by means of (10) and considering that $L=N^{2} / \Re$.

\section{B. Magnetic torque calculation}

In this section, an expression for the magnetic torque that acts on the movable armature is obtained by applying an energy balance over the electromagnetic components of the relay.

The electric energy supplied to the coil, $W_{e}$, is the only energy input of the balance. This energy is transformed in electromagnetic energy, $W_{e m}$, mechanical work, $W_{m}$, and energy losses, $W_{l}$. Hence, the balance can be expressed as

$$
W_{e}=W_{e m}+W_{m}+W_{l},
$$

or, in differential form, as

$$
\mathrm{d} W_{e}=\mathrm{d} W_{e m}+\mathrm{d} W_{m}+\mathrm{d} W_{l} .
$$

In the first place, the electric energy supplied to the system in a differential time $\mathrm{d} t$ can be calculated as

$$
\mathrm{d} W_{e}=v i \mathrm{~d} t .
$$

By substituting (1) in the previous expression, it becomes

$$
\mathrm{d} W_{e}=\left(R i+N \frac{\mathrm{d} \phi}{\mathrm{d} t}\right) i \mathrm{~d} t=R i^{2} \mathrm{~d} t+N i \mathrm{~d} \phi,
$$

and, by using (2),

$$
\mathrm{d} W_{e}=R i^{2} \mathrm{~d} t+\phi \Re \mathrm{d} \phi .
$$

On the second place, the total electromagnetic energy of a system of volume $V$ can be calculated as

$$
W_{e m}=\frac{1}{2} \iiint_{V} \mathbf{B} \cdot \mathbf{H} \mathrm{d} V,
$$

where $\mathbf{B}$ and $\mathbf{H}$ are, respectively, the magnetic flux density and the magnetic field intensity vectors, defined for every point in the space. Magnetic equivalent circuits are based on assuming a three-dimensional electromagnetic system as onedimensional, being the closed path followed by the magnetic 
flux (see Fig. 2(a)) the dominant dimension. Let $l$ be the position variable that defines the main path of the flux and let $\hat{\mathbf{u}}_{l}(l)$ be the unit vector in the direction of $l$. According to this simplification, $\mathbf{B}$ and $\mathbf{H}$ depend only on $l$ and are in the direction of $\hat{\mathbf{u}}_{l}$, i.e., $\mathbf{B}(l)=B(l) \hat{\mathbf{u}}_{\boldsymbol{l}}(l)$ and $\mathbf{H}(l)=H(l) \hat{\mathbf{u}}_{l}(l)$, where $B=\|\mathbf{B}\|$ and $H=\|\mathbf{H}\|$. Additionally, let $A(l)$ be the cross-sectional area of the magnetic circuit in the position $l$ and let the differential volume $\mathrm{d} V$ be defined according to this area as $\mathrm{d} V=A(l) \mathrm{d} l$. Hence, (17) may be rewritten as

$$
W_{\text {em }}=\frac{1}{2} \oint B(l) H(l) A(l) \mathrm{d} l .
$$

It also follows from the previous simplification that the magnetic flux in a magnetic circuit, which is defined as the integral of $\mathbf{B}$ over the cross-section, can be calculated for a given position $l$ as

$$
\phi=\iint_{A(l)} \mathbf{B} \cdot \mathrm{d} \mathbf{s}=\iint_{A(l)} B(l) \mathrm{d} s=B(l) A(l) .
$$

Under the assumption that there is no leakage flux, i.e.,

$$
\oiint_{S_{V}} \mathbf{B} \cdot \mathrm{d} \mathbf{s}=0
$$

where $S_{V}$ is the external surface of the magnetic circuit, the magnetic flux becomes independent of $l$. Then, given (19), (18) transforms into

$$
W_{e m}=\frac{1}{2} \phi \oint H(l) \mathrm{d} l,
$$

and, according to Ampere's law, i.e., $\oint H(l) \mathrm{d} l=N i$, into

$$
W_{\text {em }}=\frac{1}{2} \phi N i \text {. }
$$

The previous equation may now be expressed in terms of the current through the coil or of the flux through the magnetic circuit by using (2).

$$
W_{\text {em }}=\frac{1}{2} \frac{N^{2} i^{2}}{\Re}=\frac{1}{2} \phi^{2} \Re .
$$

Note that precisely one of these expressions is obtained if inductance $L$ of the coil is firstly calculated as $L=N^{2} / \Re$ and then the expression for the energy stored in an inductor is used.

$$
W_{\text {em }}=\frac{1}{2} L i^{2}=\frac{1}{2} \frac{N^{2}}{\Re} i^{2} .
$$

By using the form in terms of $\phi$, the second term of the balance is finally obtained.

$$
\mathrm{d} W_{e m}=\frac{1}{2} \phi^{2} \mathrm{~d} \Re+\phi \Re \mathrm{d} \phi .
$$

In the third place, differential mechanical work $\mathrm{d} W_{m}$ is created by the electromagnetic torque $\tau_{m a g}$ that acts on the movable armature when it rotates a differential angle $\mathrm{d} \theta_{1}$.

$$
\mathrm{d} W_{m}=\tau_{m a g} \mathrm{~d} \theta_{1} .
$$

Finally, the fourth term of the balance is obtained by considering that the only losses in the electromagnetic components are due to the Joule heating effect in the coil resistance.

$$
\mathrm{d} W_{l}=R i^{2} \mathrm{~d} t .
$$

Thus, by substituting each term in the energy balance,

$$
R i^{2} \mathrm{~d} t+\phi \Re \mathrm{d} \phi=\frac{1}{2} \phi^{2} \mathrm{~d} \Re+\phi \Re \mathrm{d} \phi+\tau_{m a g} \mathrm{~d} \theta_{1}+R i^{2} \mathrm{~d} t .
$$

The previous equation can now be solved to obtain the magnetic torque $\tau_{m a g}$ applied to the movable armature.

$$
\tau_{m a g}=-\frac{1}{2} \phi^{2} \frac{\mathrm{d} \Re}{\mathrm{d} \theta_{1}} .
$$

By deriving and substituting (10) in (29), the final expression for $\tau_{m a g}$ is obtained.

$$
\tau_{m a g}=-\frac{1}{2} \phi^{2} k_{1}=-\frac{1}{2} \phi^{2} \frac{r_{g}}{\mu_{0} A_{g}} .
$$

Note that the minus sign, together with the squared value of $\phi$, indicates that the torque acts always in the opposite direction of $\theta_{1}$.

\section{MECHANICAL MODEL}

To describe the motion of the relay we propose the planar mechanism of Fig. 3. This mechanical model is composed

\begin{tabular}{|c|c|}
\hline$x_{P}, y_{P}$ & $\begin{array}{l}\text { Coordinates of point } P \text { in the } \mathrm{XY} \text { coordi- } \\
\text { nate system. }\end{array}$ \\
\hline$\vec{r}_{P}=\left(\begin{array}{l}x_{P} \\
y_{P}\end{array}\right.$ & Position vector of point $P$. \\
\hline $\overrightarrow{P Q}$ & Vector from point $P$ to point $Q$. \\
\hline$P Q=\|\overrightarrow{P Q}\|$ & Length of vector $\overrightarrow{P Q}$ \\
\hline$g \quad \| \quad 1$ & Gravity. \\
\hline$G_{i}$ & Center of mass of rigid body $i$. \\
\hline$m_{i}$ & Mass of rigid body $i$. \\
\hline$I_{i}$ & $\begin{array}{l}\text { Moment of inertia of rigid body } i \text { in the } \\
\text { XY plane. }\end{array}$ \\
\hline$\dot{\theta}, \ddot{\theta}$ & First, second time derivative of $\theta$. \\
\hline
\end{tabular}
of four rigid bodies connected by joints and numbered in circles in the figure. Body 1 models the movable armature and body 2 corresponds to the plastic pusher. Since the movable contact is made from a flexible copper sheet, it is modeled as the union of two rigid bodies, 3 and 4, which are connected by joint $E$. Spring torques that model the sheet rigidity are included in joints $D$ and $E$. An actual torsion spring that exists in the bottom of the movable armature is also included in the mechanical model in joint $A$. To model the friction of the whole mechanism, a viscous friction force is included in joint $H$. Before analyzing the mechanism we introduce the notation for this section:

Joints $A, D$ and $H$ are fixed and have known positions. If $A$ is selected as the origin of the coordinate system, then

$$
\vec{r}_{A}=\left(\begin{array}{c}
0 \\
0
\end{array}\right), \quad \vec{r}_{H}=\left(\begin{array}{c}
x_{H} \\
y_{H}
\end{array}\right), \quad \vec{r}_{D}=\left(\begin{array}{c}
x_{D} \\
y_{D}
\end{array}\right) .
$$




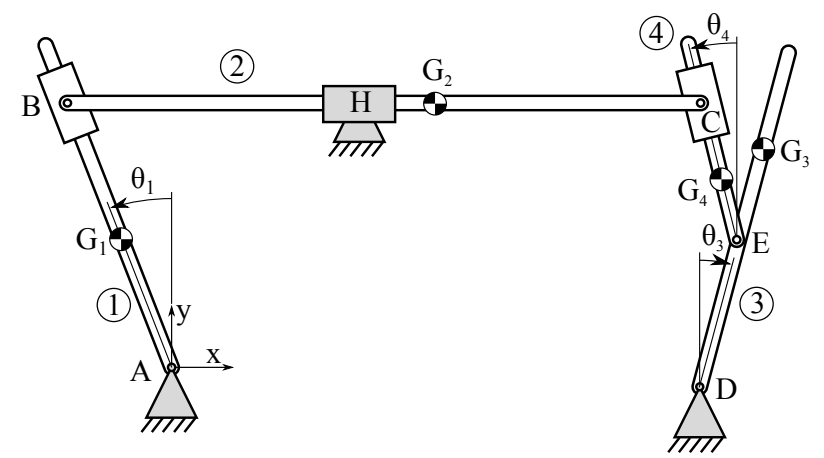

Fig. 3. Mechanical model.

The position of the rest of the points of the mechanism depends on angular variables $\theta_{1}, \theta_{3}$ and $\theta_{4}$. Considering that $A G_{1}, B C, B G_{2}, D E, D G_{3}$, and $E G_{4}$ are known constant lengths of the mechanism, the following vectors of the kinematic chain are calculated:

$$
\begin{array}{ll}
\overrightarrow{A G_{1}}=\left(\begin{array}{c}
-A G_{1} \sin \left(\theta_{1}\right) \\
A G_{1} \cos \left(\theta_{1}\right)
\end{array}\right), & \overrightarrow{A B}=\left(\begin{array}{c}
-y_{H} \tan \left(\theta_{1}\right) \\
y_{H}
\end{array}\right), \\
\overrightarrow{B G_{2}}=\left(\begin{array}{c}
B G_{2} \\
0
\end{array}\right), & \overrightarrow{B C}=\left(\begin{array}{c}
B C \\
0
\end{array}\right), \\
\overrightarrow{D E}=\left(\begin{array}{c}
D E \sin \left(\theta_{3}\right) \\
D E \cos \left(\theta_{3}\right)
\end{array}\right), & \overrightarrow{D G_{3}}=\left(\begin{array}{c}
D G_{3} \sin \left(\theta_{3}\right) \\
D G_{3} \cos \left(\theta_{3}\right)
\end{array}\right), \\
\overrightarrow{E G_{4}}=\left(\begin{array}{c}
-E G_{4} \sin \left(\theta_{4}\right) \\
E G_{4} \cos \left(\theta_{4}\right)
\end{array}\right) . &
\end{array}
$$

Then, by adding and subtracting these vectors, the position of centers of mass $G_{1}, G_{2}, G_{3}$ and $G_{4}$ and joints $B, C$ and $E$ can be calculated as

$$
\begin{array}{ll}
\vec{r}_{G 1}=\overrightarrow{A G_{1}}, & \vec{r}_{B}=\overrightarrow{A B}, \\
\vec{r}_{G 2}=\overrightarrow{A B}+\overrightarrow{B G_{2}}, & \vec{r}_{C}=\overrightarrow{A B}+\overrightarrow{B C}, \\
\vec{r}_{G 3}=\vec{r}_{D}+\overrightarrow{D G_{3}}, & \vec{r}_{E}=\vec{r}_{D}+\overrightarrow{D E}, \\
\vec{r}_{G 4}=\vec{r}_{D}+\overrightarrow{D E}+\overrightarrow{E G_{4}} . &
\end{array}
$$

Note that, although the previous equations are in terms of $\theta_{1}, \theta_{3}$ and $\theta_{4}$, the mechanism has only two degrees of freedom because the variables are intrinsically related.

$$
\theta_{4}=\operatorname{atan}\left(\frac{x_{E}\left(\theta_{3}\right)-x_{C}\left(\theta_{1}\right)}{y_{C}\left(\theta_{1}\right)-y_{E}\left(\theta_{3}\right)}\right) .
$$

Free body diagrams of the four components of the mechanism are presented in Fig. 4. All the internal and external forces and torques that act on the bodies are represented in these diagrams by means of arrows, each pointing to the direction which has been defined as positive. Revolute joints $A, D$ and $E$ generate internal forces of unknown direction which can be decomposed in the $\mathrm{X}$ and $\mathrm{Y}$ coordinates, obtaining $F_{A x}$, $F_{A y}, F_{D x}, F_{D y}, F_{E x}$ and $F_{E y}$. Pin-slot joints $B$ and $C$ create forces $F_{B}$ and $F_{C}$, which are respectively perpendicular to the direction of solids 1 and 3 , and prismatic joint $H$ creates

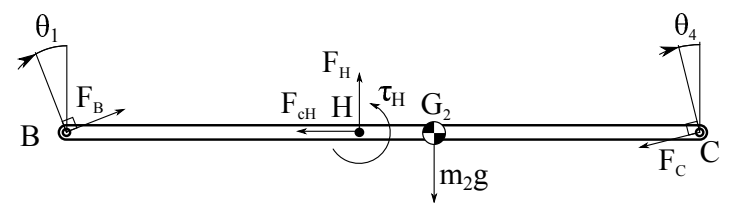

(a) Body 2 .

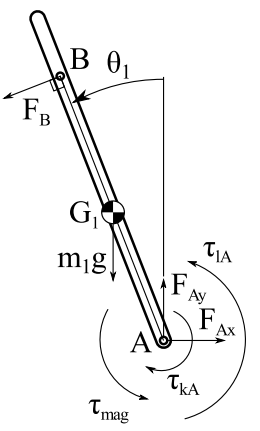

(b) Body 1 .

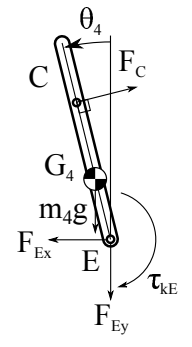

(c) Body 4 .

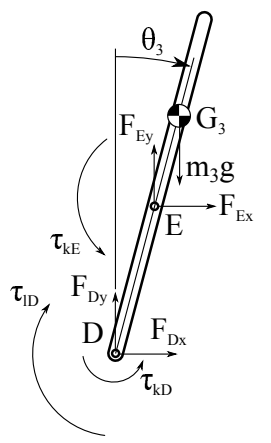

(d) Body 3 .
Fig. 4. Free body diagrams.

a force $F_{H}$ in the $\mathrm{Y}$ coordinate and a torque $\tau_{H}$ in the $\mathrm{Z}$ coordinate.

Spring torques $\tau_{k_{A}}, \tau_{k_{D}}$ and $\tau_{k_{E}}$ in joints $A, D$ and $E$ are calculated by means of Hooke's law:

$$
\begin{aligned}
& \tau_{k_{A}}=k_{A}\left(\theta_{1}-\theta_{1,0}\right), \\
& \tau_{k_{D}}=k_{D}\left(\theta_{3}-\theta_{3,0}\right), \\
& \tau_{k_{E}}=k_{E}\left(\theta_{E}-\theta_{E, 0}\right), \quad \theta_{E}=\theta_{3}+\theta_{4},
\end{aligned}
$$

where $k_{A}, k_{D}$ and $k_{E}$ are stiffness constants and $\theta_{1,0}, \theta_{3,0}$ and $\theta_{E, 0}$ are the springs natural angles. With regard to viscous friction force $F_{c H}$, it is calculated as

$$
F_{c H}=c_{H} \dot{x}_{G_{2}},
$$

where $c_{H}$ is a constant damping coefficient.

The motion of the mechanism is restricted to specific ranges in variables $\theta_{1}$ and $\theta_{3}$ due to the position of the fixed armature and the contacts (Fig. 1). To model these limits, torques $\tau_{l A}$ and $\tau_{l D}$ act in joints $A$ and $D$ when bodies 1 and 3 are beyond their boundaries. Besides of limiting the motion, these torques also model the bounces generated when the moving parts hit the fixed ones. This is achieved by means of torsional springdamper systems which emulate the Kelvin-Voigt viscoelastic model [12]. Considering $\left[\theta_{1, \min }, \theta_{1, \max }\right]$ and $\left[\theta_{3, \min }, \theta_{3, \max }\right]$ the ranges of $\theta_{1}$ and $\theta_{3}, \tau_{l A}$ and $\tau_{l D}$ are given by the following expressions:

$$
\begin{gathered}
\tau_{l A}=\left\{\begin{aligned}
-k_{l A}\left(\theta_{1}-\theta_{1, \text { min }}\right)-c_{l A} \dot{\theta_{1}}, & \text { if } \theta_{1}<\theta_{1, \text { min }} \\
-k_{l A}\left(\theta_{1}-\theta_{1, \text { max }}\right)-c_{l A} \dot{\theta_{1},} & \text { if } \theta_{1}>\theta_{1, \text { max }} \\
0, & \text { otherwise, }
\end{aligned}\right. \\
\tau_{l D}=\left\{\begin{aligned}
-k_{l D}\left(\theta_{3}-\theta_{3, \text { min }}\right)-c_{l D} \dot{\theta_{3},} & \text { if } \theta_{3}<\theta_{3, \text { min }} \\
-k_{l D}\left(\theta_{3}-\theta_{3, \text { max }}\right)-c_{l D} \dot{\theta_{3},}, & \text { if } \theta_{3}>\theta_{3, \text { max }} \\
0, & \text { otherwise, }
\end{aligned}\right.
\end{gathered}
$$


where $k_{l A}$ and $k_{l D}$ are the stiffness constants and $c_{l A}$ and $c_{l D}$ the damping coefficients of the spring-damping torques.

The weights of the four bodies and magnetic torque $\tau_{\text {mag }}$, which acts on body 1 and is given by the electromagnetic model, are also included in the mechanism. Equations for linear and angular accelerations are then obtained by applying Newton's second law. Three equations are presented below for each body, corresponding to the net forces in coordinates $\mathrm{X}$ and $\mathrm{Y}$ and the net torque in coordinate $\mathrm{Z}$. For bodies 1 to 4 , torques have been respectively calculated in points $A, B, D$ and $E$.

Equations for body 1:

$F_{A x}-F_{B} \cos \left(\theta_{1}\right)=m_{1} \ddot{x}_{G_{1}}$,

$F_{A y}-F_{B} \sin \left(\theta_{1}\right)-m_{1} g=m_{1} \ddot{y}_{G_{1}}$,

$F_{B} A B+m_{1} g A G_{1} \sin \left(\theta_{1}\right)+\tau_{l A}-\tau_{k_{A}}+\tau_{m a g}=I_{1} \ddot{\theta}_{1}$.

Equations for body 2:

$F_{B} \cos \left(\theta_{1}\right)-F_{C} \cos \left(\theta_{4}\right)-F_{c_{H}}=m_{2} \ddot{x}_{G_{2}}$,

$F_{B} \sin \left(\theta_{1}\right)+F_{H}-m_{2} g-F_{C} \sin \left(\theta_{4}\right)=m_{2} \ddot{y}_{G_{2}}$,

$F_{H} B H-m_{2} g B G_{2}-F_{C} B C \sin \left(\theta_{4}\right)+\tau_{H}=0$.

Equations for body 3 :

$$
\begin{aligned}
& F_{D x}+F_{E x}=m_{3} \ddot{x}_{G_{3}}, \\
& F_{D y}+F_{E y}-m_{3} g=m_{3} \ddot{y}_{G_{3}}, \\
& F_{E y} D E \sin \left(\theta_{3}\right)-F_{E x} D E \cos \left(\theta_{3}\right) \\
& \quad-m_{3} g D G_{3} \sin \left(\theta_{3}\right)+\tau_{k_{D}}+\tau_{k_{E}}-\tau_{l D}=-I_{3} \ddot{\theta}_{3} .
\end{aligned}
$$

Equations for body 4 :

$$
\begin{aligned}
& F_{C} \cos \left(\theta_{4}\right)-F_{E x}=m_{4} \ddot{x}_{G_{4}}, \\
& F_{C} \sin \left(\theta_{4}\right)-F_{E y}-m_{4} g=m_{4} \ddot{y}_{G_{4}}, \\
& m_{4} g E G_{4} \sin \left(\theta_{4}\right)-F_{C} E C-\tau_{k_{E}}=I_{4} \ddot{\theta}_{4} .
\end{aligned}
$$

By substituting expressions (31) to (37) as well as the position of the centers of mass, the system of equations (38) to (49), which has 12 unknown variables $\left(F_{A x}, F_{A y}, F_{B}\right.$, $F_{H}, \tau_{H}, F_{C}, F_{D x}, F_{D y}, F_{E x}, F_{E y}, \theta_{1}$ and $\left.\theta_{3}\right)$, permits the resolution of the mechanism.

\section{Model VALIDation}

\section{A. Implementation for simulation}

The electromechanical model of the relay has been implemented in MATLAB Simulink to carry out simulations and to prove its validity. The electromagnetic equations have been written in MATLAB code and included in Simulink by means of a MATLAB Function block. On the other hand, the mechanical part has been implemented using blocks of the SimMechanics library. This tool, apart from resolving the equations for the motion of the model, provides a graphical interface which allows a 3D visualization of the system during the simulation. Fig. 5 shows some images of the developed model in the SimMechanics graphical interface.

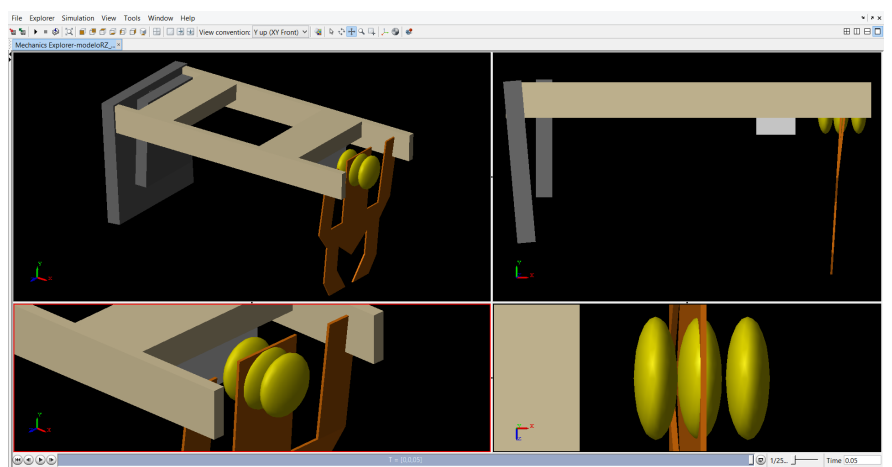

Fig. 5. SimMechanics graphical interface.

\section{B. Experimental tests}

An electronic circuit (Fig. 6), similar to those used for relay switching in industry applications, has been designed and implemented to conduct experimental tests on the device and validate the model. Two BJT transistors and a resistor network permit the relay to be activated and deactivated by a digital control signal, control. A general purpose diode is included for coil suppression, i.e., to limit the elevated induced voltages that appear when switching off the relay. In addition, a shunt resistor, $R_{s}$, allows for measuring the current through the coil. On the other side of the relay, two resistors and two LEDs act as power circuit and provide a simple visualization of the status of each connection. The supply voltage of the test bench, $V_{d c}$, is provided by a laboratory power source and can be adjusted from 0 up to $30 \mathrm{~V}$. Voltage and current through the coil are measured by means of a PicoScope 4824 USB oscilloscope during the tests, and voltage measurements across the normally closed and the normally open connections are also obtained throughout the experiments. All measurements are registered and stored in a personal computer. In particular, the voltage measurements from each power connection are processed and transformed for simplicity into binary data, obtaining a ' 1 ' when the voltage is nearly 0 volts, i.e., when the connection is closed, and a ' 0 ' when it is nearly 5 volts, i.e., when the connection is open. The control signal for the experiments, control, is commanded from the PC and generated by a built-in arbitrary waveform generator also included in the oscilloscope.

Several tests at different supply voltages and using different control signals have been performed on the relay by means of this test bench. Data from a first set of experiments have been used to adjust some of the model parameters which were partially undetermined. To be coherent, the results presented in this section correspond to a second set, different from the one used for the adjustment. Specifically, experimental data from two different tests at supply voltages of 24 and $30 \mathrm{~V}$ are presented in this paper to prove the validity of the model. The control signal of both tests consisted of a 25 milliseconds 'on' period followed by a 25 milliseconds 'off' period (Fig. 7). The voltage across the coil registered during the experiments, Figs. 8 and 12, has been used as input of the model for the corresponding simulations. The comparison 


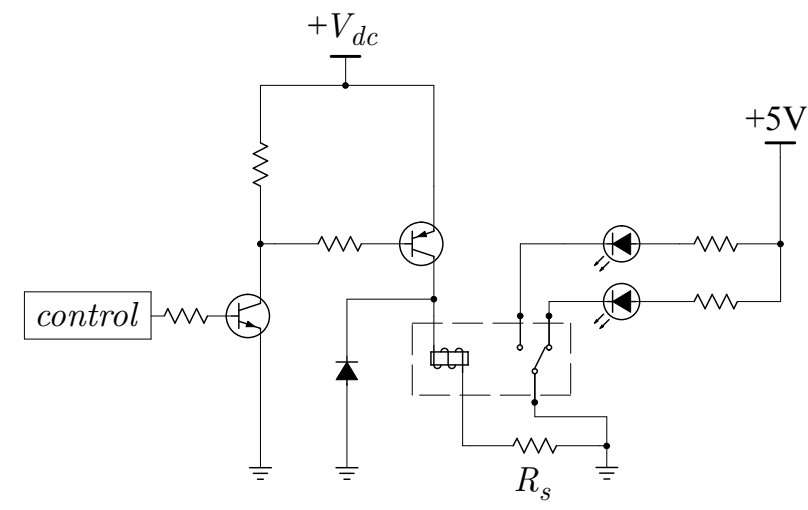

Fig. 6. Circuit diagram of the experimental test bench. Shunt resistor $R_{s}$ permits the measurement of the current through the coil.

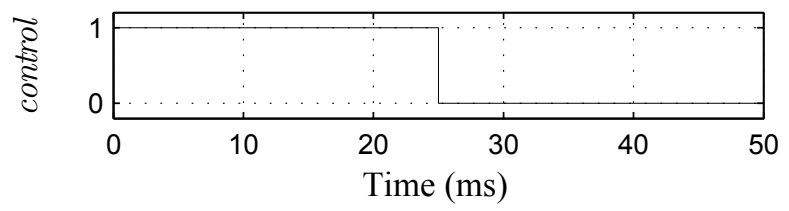

Fig. 7. Control signal used in the experimental tests.

between the experimental and the simulated outputs of the system is presented and discussed in the following paragraphs and figures.

In the first place, Fig. 9 shows a comparison between the experimental coil current during the $24 \mathrm{~V}$ test and the current obtained from simulation. The nonlinear dynamics of the system is evident during the closure of the armature (first 25 milliseconds) and also in the opening (last 25 milliseconds). The current predicted by the model fits well with the experimental data. For the same test, Figs. 10 and 11 show, respectively, the status of the normally closed and the normally open contacts, NCC and NOC. During the closure, it can be seen that the movable contact does not leave its normal position immediately after the activation of the control signal, but it takes about 7.2 milliseconds. This behavior is due to the dynamics of the magnetic torque, which is lower than the elastic forces until a given value of the current through the coil is reached (30). Approximately 2.5 milliseconds later, the movable contact hits the normally open contact, causing some bounces. Altogether, the relay takes about 10 milliseconds to perform the complete breaking process, i.e., to open the normally closed connection and close the normally open connection. The behavior predicted by the model in this sense fits almost exactly with the measurements. The procedure is reversed during the opening, causing the movable contact to return to its original position, opening the normally open connection and closing the normally closed connection. In this process, the time needed to begin the motion is about 9 milliseconds, the free motion lasts about 1 millisecond and the bounces take approximately 2.5 milliseconds. It is shown again that the prediction given by the model agrees very well with the measurements.
The results from the test with the supply voltage of $30 \mathrm{~V}$ are presented in Figs. 13 to 15. Although the system dynamics is similar to that of the $24 \mathrm{~V}$ test, there are some differences due to the nonlinearities, specially during the closure. At this stage, the current through the coil reaches the steady state in about 10 milliseconds, faster than in the $24 \mathrm{~V}$ experiment. Nonetheless, its behavior during the opening is more similar to the previous test. Similar conclusions are drawn from analyzing the measurements of the contacts. It can be seen that the closure is faster, mainly because the motion is started earlier, although the period of bounces is slightly longer. The opening is however a little slower because of the higher current in the closure, which generates a more elevated magnetic torque (30) that takes more time to be overtaken by the elastic forces. In spite of these differences, the model provides a very good estimation of the relay dynamics also for this experiment.

Finally, tables I and II summarize the duration of the main stages of the motion during the closure and the opening. The process has been divided into three time periods: the time needed to start the motion, the period of free motion and the period of bounces. The sum of these three time intervals results in the total making or breaking duration. The tables present the results from both experimental tests and also from the respective simulations. The agreement between model and reality is proved once again.

\section{Additional considerations}

One of the main advantages of MEC-based models with respect to FEM models is their very low computational requirements. To illustrate this statement and compare both methods, a FEM model, Fig. 16, of the electromagnetic switch investigated in the paper has been implemented and some simulations have been performed. Like the MEC model, this model also includes the magnetic saturation of the core. However, given that a complete transient and nonlinear simulation would need a very high computational time, only the electromagnetism of the system has been implemented, i.e., the position of the mechanism is given as input, and only stationary analyses have been performed. The results are nevertheless really convincing. While a stationary simulation of the FEM model takes more than 15 minutes on a 4 th generation Intel Core i7 computer, a dynamic 50 milliseconds simulation on the same PC takes only 15 seconds using our model. Although no transient simulations have been performed with the FEM model, the results obtained show clearly the great difference between methods.

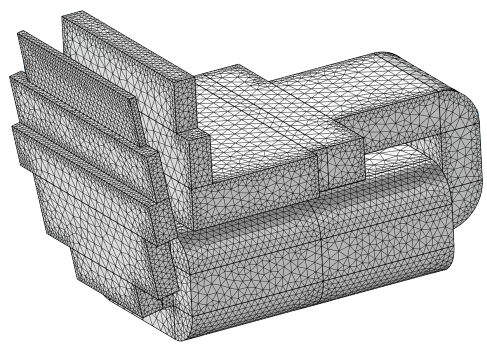

Fig. 16. FEM model of the electromagnetic relay. 


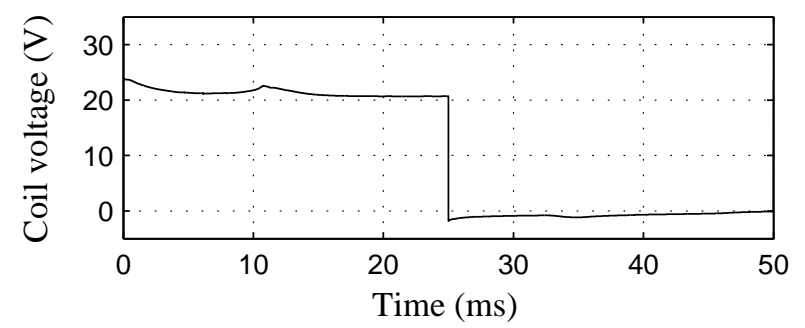

Fig. 8. Measured voltage across the coil. $V_{d c}=24 \mathrm{~V}$. This voltage profile is used as input for the corresponding simulation.

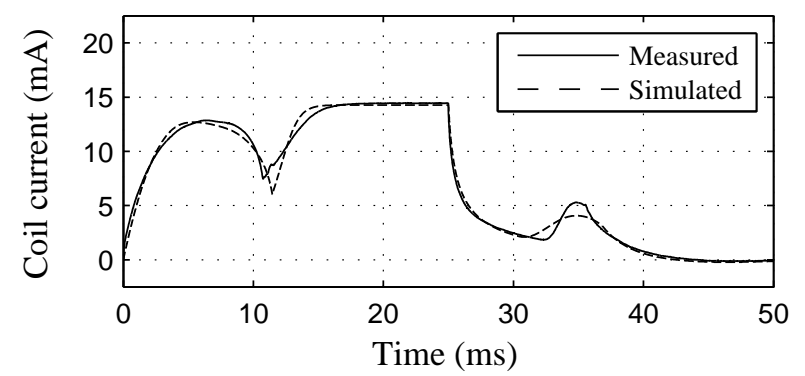

Fig. 9. Measured and simulated coil current. $V_{d c}=24 \mathrm{~V}$.
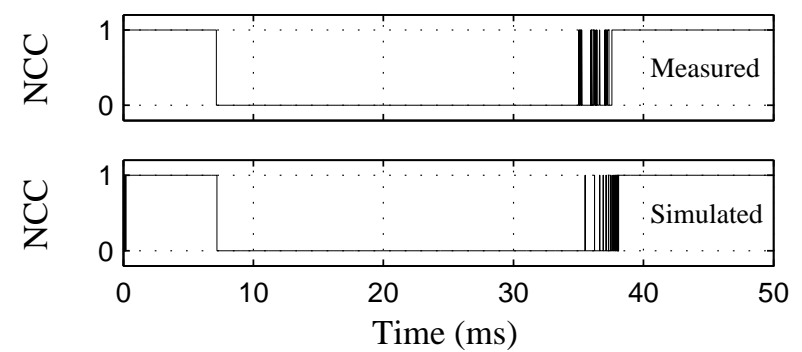

Fig. 10. Measured and simulated normally closed contact (NCC). $V_{d c}=24 \mathrm{~V}$. ' 0 ' means open connection and ' 1 ' means closed connection.
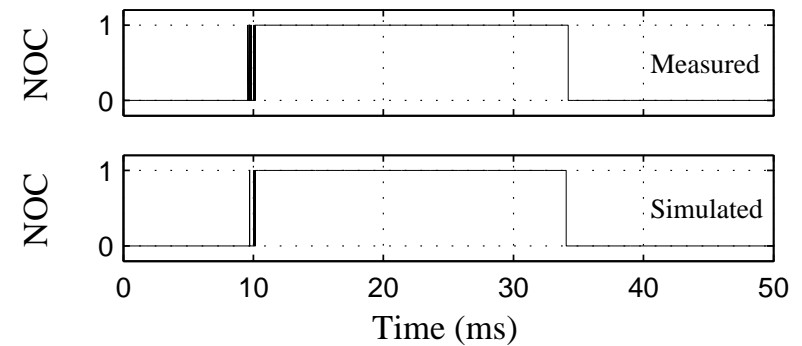

Fig. 11. Measured and simulated normally open contact (NOC). $V_{d c}=24 \mathrm{~V}$. ' 0 ' means open connection and ' 1 ' means closed connection.

TABLE I. MOTION STAGES DURING THE ClOSURE.

\begin{tabular}{clccc}
\hline \multicolumn{2}{c}{ Test } & $\begin{array}{c}\text { Motion start } \\
(\mathrm{ms})\end{array}$ & $\begin{array}{c}\text { Free motion } \\
(\mathrm{ms})\end{array}$ & $\begin{array}{c}\text { Bounces } \\
(\mathrm{ms})\end{array}$ \\
\hline \multirow{2}{*}{$V_{d c}=24 \mathrm{~V}$} & Measured & 7.16 & 2.43 & 0.57 \\
& Simulated & 7.20 & 2.51 & 0.45 \\
\hline \multirow{2}{*}{$V_{d c}=30 \mathrm{~V}$} & Measured & 4.72 & 1.15 & 0.65 \\
& Simulated & 4.91 & 1.08 & 0.60 \\
\hline
\end{tabular}

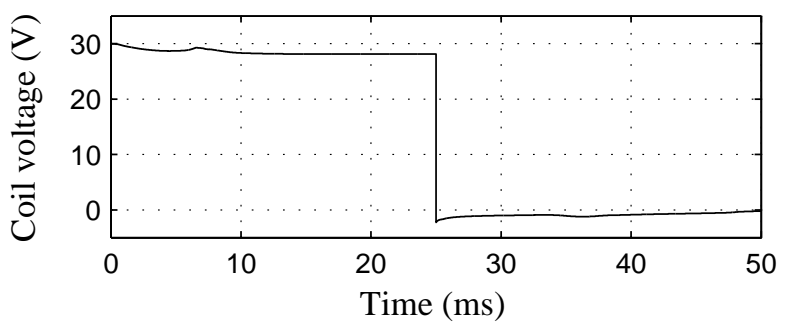

Fig. 12. Measured voltage across the coil. $V_{d c}=30 \mathrm{~V}$. This voltage profile is used as input for the corresponding simulation.

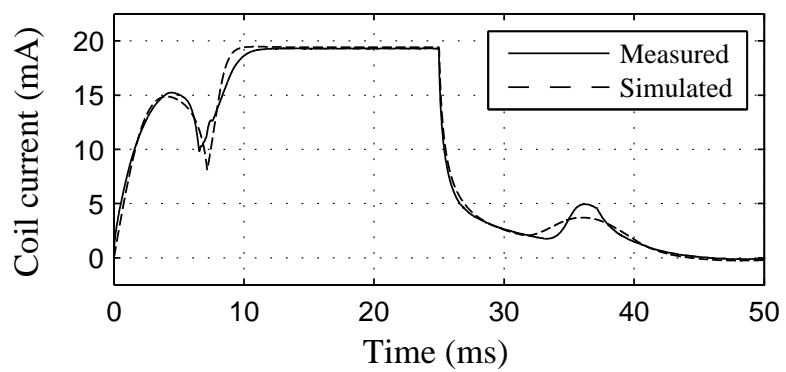

Fig. 13. Measured and simulated coil current. $V_{d c}=30 \mathrm{~V}$.
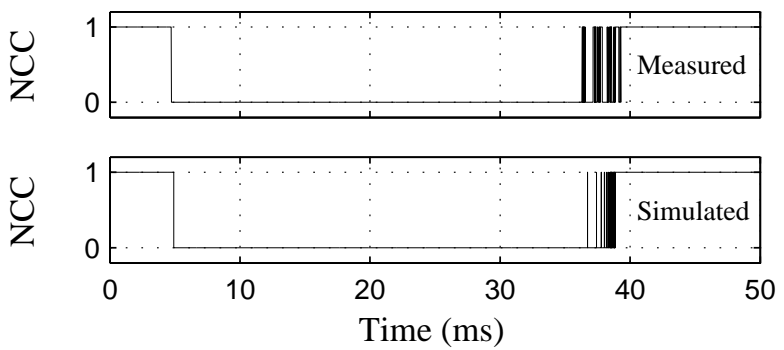

Fig. 14. Measured and simulated normally closed contact (NCC). $V_{d c}=30 \mathrm{~V}$. ' 0 ' means open connection and ' 1 ' means closed connection.

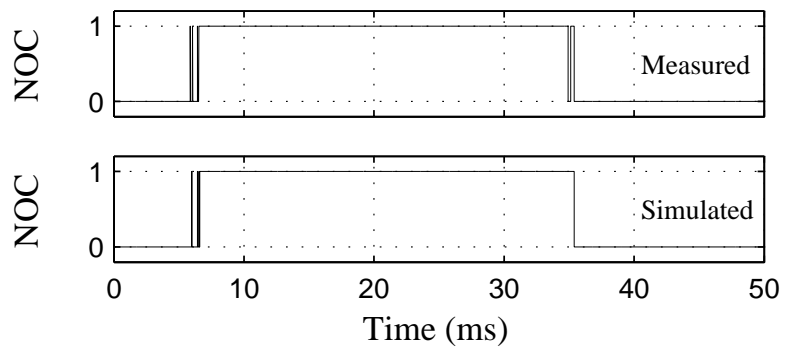

Fig. 15. Measured and simulated normally open contact (NOC). $V_{d c}=30 \mathrm{~V}$. ' 0 ' means open connection and ' 1 ' means closed connection.

TABLE II. MOTION STAGES DURING THE OPENING.

\begin{tabular}{|c|c|c|c|c|}
\hline \multicolumn{2}{|c|}{ Test } & $\begin{array}{l}\text { Motion start } \\
\text { (ms) }\end{array}$ & $\begin{array}{l}\text { Free motion } \\
(\mathrm{ms})\end{array}$ & $\begin{array}{c}\text { Bounces } \\
(\mathrm{ms})\end{array}$ \\
\hline \multirow{2}{*}{$V_{d c}=24 \mathrm{~V}$} & Measured & 9.22 & 0.82 & 2.54 \\
\hline & Simulated & 9.06 & 1.44 & 2.58 \\
\hline \multirow{2}{*}{$V_{d c}=30 \mathrm{~V}$} & Measured & 9.93 & 1.39 & 2.97 \\
\hline & Simulated & 10.39 & 1.33 & 2.12 \\
\hline
\end{tabular}




\section{CONCLUSIONS}

A new model for predicting the electromagnetic and motion dynamics of electromechanical relays has been developed and validated. The electromagnetic dynamics has been studied by obtaining the total reluctance of the magnetic circuit. Then, the magnetic torque that acts on the mechanism, which depends on the magnetic flux and the position of the mechanism, has been calculated by means of a balance of the electromagnetic energy stored in the system. Apart from the current and the magnetic flux, the instantaneous value of the coil inductance is also obtained. A planar mechanism with two degrees of freedom has been proposed to explain the motion of the relay, and a comprehensive theoretical description of the kinematics and dynamics of the system has been presented. Spring-damping torques based on the Kelvin-Voigt viscoelastic model have been included to restrict the motion of the mechanism and to model the bounces generated in the switchings.

Experimental tests have been carried out on the actual relay to validate the model. It has been shown that it fits correctly to the nonlinear behavior of the system, providing good estimations for the current through the coil and the motion of the mechanism. The agreement between simulated and measured data has been proved to be very high even at different supply voltages and both in the closure and the opening of the relay.

The developed model, since it is based on a magnetic equivalent circuit, is much less time consuming than FEM based models. This feature makes it very appropriate for processes needing a great amount of simulations, e.g., the optimization of new control algorithms. In addition, since it is fully parametrized, temperature dependence or stochastic behaviors of the parameters could be easily added to the model to improve its performance or to carry out sensitivity analyses. Note that this feature may be especially helpful, e.g., when designing and validating robust or adaptive controllers.

\section{ACKNOWLEDGMENT}

This work was partially supported by projects IPT-20111158-920000 of subprogram INNPACTO and RTC-20141847-6 of subprogram RETOS-COLABORACIÓN from Ministerio de Economía y Competitividad, Gobierno de España / European Union and by grant FPU14/04171 from Ministerio de Educación, Cultura y Deporte, Gobierno de España.

\section{REFERENCES}

[1] P. Barkan, "A study of the contact bounce phenomenon," IEEE Trans. Power App. Syst., no. 2, pp. 231-240, 1967.

[2] J. W. McBride, "Electrical contact bounce in medium-duty contacts," IEEE Trans. Compon. Hybrids, Manuf. Technol., vol. 12, no. 1, pp. 82-90, 1989.

[3] T. S. Davies, H. Nouri, and F. W. Britton, "Towards the control of contact bounce," IEEE Trans. Compon. Packag. Manuf. Technol., vol. 19 , no. 3, pp. 353-359, 1996.

[4] H. Nouri, N. Larsen, and T. Davies, "Contact bounce simulation using matlab," in Proc. 43rd IEEE Holm Conf. Elect. Contacts. IEEE, 1997, pp. $284-288$.

[5] M. Amrhein and P. T. Krein, "3-d magnetic equivalent circuit framework for modeling electromechanical devices," IEEE Trans. Energy Convers., vol. 24, no. 2, pp. 397-405, 2009.
[6] J. R. Riba Ruiz and A. Garcia Espinosa, "A novel parametric model for ac contactors," IEEE Trans. Magn., vol. 44, no. 9, pp. 2215-2218, 2008.

[7] A. García Espinosa, J. R. Riba Ruiz, and X. Alabern Morera, "A sensorless method for controlling the closure of a contactor," IEEE Trans. Magn., vol. 43, no. 10, pp. 3896-3903, 2007.

[8] H. Lin, X. Wang, S. Fang, P. Jin, and S. Ho, "Design, optimization, and intelligent control of permanent-magnet contactor," IEEE Trans. Ind. Electron., vol. 60, no. 11, pp. 5148-5159, 2013.

[9] Z. Guofo, W. Qiya, and R. Wanbin, "An output space-mapping algorithm to optimize the dimensional parameter of electromagnetic relay," IEEE Trans. Magn., vol. 47, no. 9, pp. 2194-2199, 2011.

[10] D. Wattiaux and O. Verlinden, "Modelling of the dynamic behaviour of electromechanical relays for the analysis of sensitivity to shocks and vibrations," Exp. Mech., vol. 51, no. 9, pp. 1459-1472, 2011.

[11] Z. Guofu, W. Qiya, Y. Wenying, and L. Huimin, "Permanent-magnet equivalent model of calculating relay's static attractive torque characteristics by finite element method," IEEE Trans. Magn., vol. 48, no. 9, pp. 2467-2471, 2012.

[12] X. Jun, H. Jun-jia, and Z. Chun-yan, "A dynamic model of electromagnetic relay including contact bounce," in 2008 Int. Conf. Elect. Mach. and Syst. IEEE, 2008, pp. 4144-4149.

[13] X. Wang, H. Lin, S. Ho, S. Fang, and P. Jin, "Analysis of dynamic characteristics of permanent magnet contactor with sensorless displacement profile control," IEEE Trans. Magn., vol. 46, no. 6, pp. 1633-1636, 2010.

[14] P. M. dos Santos Dias de Moraes and A. J. Perin, "An electronic control unit for reducing contact bounce in electromagnetic contactors," IEEE Trans. Ind. Electron., vol. 55, no. 2, pp. 861-870, 2008.

[15] K. Austin and J. Wagner, "Development of an electromagnet excited mass-pendulum system modeling and control laboratory experimenttheory and test," in European Control Conf. IEEE, 2013, pp. 256-261.

[16] S. K. Chung, C. R. Koch, and A. F. Lynch, "Flatness-based feedback control of an automotive solenoid valve," IEEE Trans. Control Syst. Technol., vol. 15, no. 2, pp. 394-401, 2007.

[17] H. Liang, J. You, X. Yong, G. Ma, and G. Zhai, "Structure classification of electromagnetic relays and comparison of typical magnetic system containing permanent magnet," in Proc. 60th IEEE Holm Conf. Elect. Contacts. IEEE, 2014, pp. 1-5.

[18] Y. Kawakami, M. Takashima, M. Hasegawa, Y. Watanabe, and K. Sawa, "An evaluation method of the contact erosion based on the dc mode test of electromagnetic contactor," in Proc. 50th IEEE Holm Conf. Elect. Contacts and 22nd Int. Conf. Elect. Contacts. IEEE, 2004, pp. 83-89.

[19] H. Liang, W. Wang, and G. Zhai, "Thermal analysis of sealed electromagnetic relays using 3-d finite element method," in Proc. 53rd IEEE Holm Conf. Elect. contacts. IEEE, 2007, pp. 262-268.

[20] L. Li, D. Ma, and Z. Li, "Cox-proportional hazards modeling in reliability analysisa study of electromagnetic relays data," IEEE Trans. Compon. Packag. Manuf. Technol., vol. 5, no. 11, pp. 1582-1589, 2015.

[21] Z. Wu, G. Wu, H. Huang, and Y. You, "A novel residual electrical endurance prediction method for low-voltage electromagnetic alternating current contactors," IEEE Trans. Compon. Packag. Manuf. Technol., vol. 5, no. 4, pp. 465-473, 2015.

[22] X. Ye, J. Deng, Y. Wang, and G. Zhai, "Quality analysis and consistency design of electromagnetic device based on approximation model," IEEE Trans. Compon. Packag. Manuf. Technol., vol. 5, no. 1, pp. 99-107, 2015.

[23] J. S. Pulskamp, R. M. Proie, and R. G. Polcawich, "Nano-and microelectromechanical switch dynamics," J. Micromech. Microeng., vol. 24, no. 1, p. 015011, 2013.

[24] E. Ramirez-Laboreo, C. Sagues, and S. Llorente, "A new model of electromechanical relays for predicting the motion and electromagnetic dynamics," in Ind. Applicat. Soc. Annu. Meet. IEEE, 2015, pp. 1-8. 